CuPAUAM. 17-1990, 9-24

\title{
EL FACTOR GRIEGO EN LA FORMACION DE LAS CULTURAS PRERROMANAS DE LA SUBMESETA SUR
}

\author{
JUAN José BLANQUez PÉREZ \\ UNIVERSIDAD AUTONOMA DE MADRID
}

\section{Resumen}

En la actualidad, gracias a la documentación arqueológica, podemos replantear el papel jugado por el "comercio griego" en el surgimiento de la cultura ibérica del Sureste de la Meseta.

Los hallazgos de Huelva, por mano focea, constituyen el punto de partida de nuestro razonamiento. Los yacimientos ibéricos de la desembocadura del Segura, en Alicante; de Los Villares (Hoya Gonzalo, Albacete); o La Boadilla (Alcaudete, Jaén), aportan la documentación necesaria para nuestra hipótesis.

La aparición de escultura fechable a principios del s. V a. de C.; de cerámica de fayenza; de silicernia, apuntan hacia un comercio griego. Con ello, la institucionalización de ritos como la libación, symposia y/o banquetes funerarios. Todos mediterráneos, pero incorporados tras un sistemático proceso de readaptación. La difusión y éxito de los materiales importados en tierras del interior demuestra la existencia de una red de comunicación en uso.

Parece evidente, pues, que sobre la población autóctona, heredera de la cultura orientalizante, se operó una última transformación acicatada por el comercio griego. El resultado, la definitiva configuración de la cultura ibérica en el sureste de la Meseta.

\section{Summary}

During the last few years, and due to the new archaeological documents, it has been possible to modify current views about the role that "Greek commerce" played in the emergence of the Iberian culture in South-East Meseta.

The Phokaian objects found in Huelva are the starting point of our reasoning. The Iberian sites at the mouth of the Segura river (Alicante) and those of Los Villares (Hoya Gonzalo, Albacete) or La Bobadilla (Alcaudete, Jaén) contribute with their materials to our hypothesis.

The appearance of sculptures dated in the beginning of the Fifth Century B. C., of fayenza ceramics, of silicernia.... all of it points to Greek commerce, and with it, to the institution of rites such as libation, symposia and/or funerary banquets; all of them Mediterranean but incorporated after a sistematic process of adaptation. The diffusion and success of imported materials in inner, non-coastal areas, proves the existence of a communication network in use. 
It seems evident that new and strong influences were exerted by Greek commerce over an auctochtonous population already transformed by oriental influences during the Orientalizing Period.

The result of these pressures was the definitive configuration of the Iberian Culture in the South-Eastern Meseta.

Hoy en día y con la perspectiva que proporciona el tiempo, al abordar el problema históricoarqueológico de las colonizaciones en la Península Ibérica y, más concretamente, al querer valorar el factor griego jugado por el mismo, observamos con curiosidad los sucesivos vaivenes sufridos en su cotización. Quizás como rechazo a temporales abusos a la hora de valorar la presencia colonial griega en la Península hemos asistido, en la última década, a una progresiva sobrevaloración de su tradicional oponente, el factor fenicio.

Un repaso a la bibliografía permite comprobarlo constatando, incluso, actitudes extremas que, en cierta manera, podrían parecer tajantes (López Domenech, 1984, 141). Un cierto "pan-

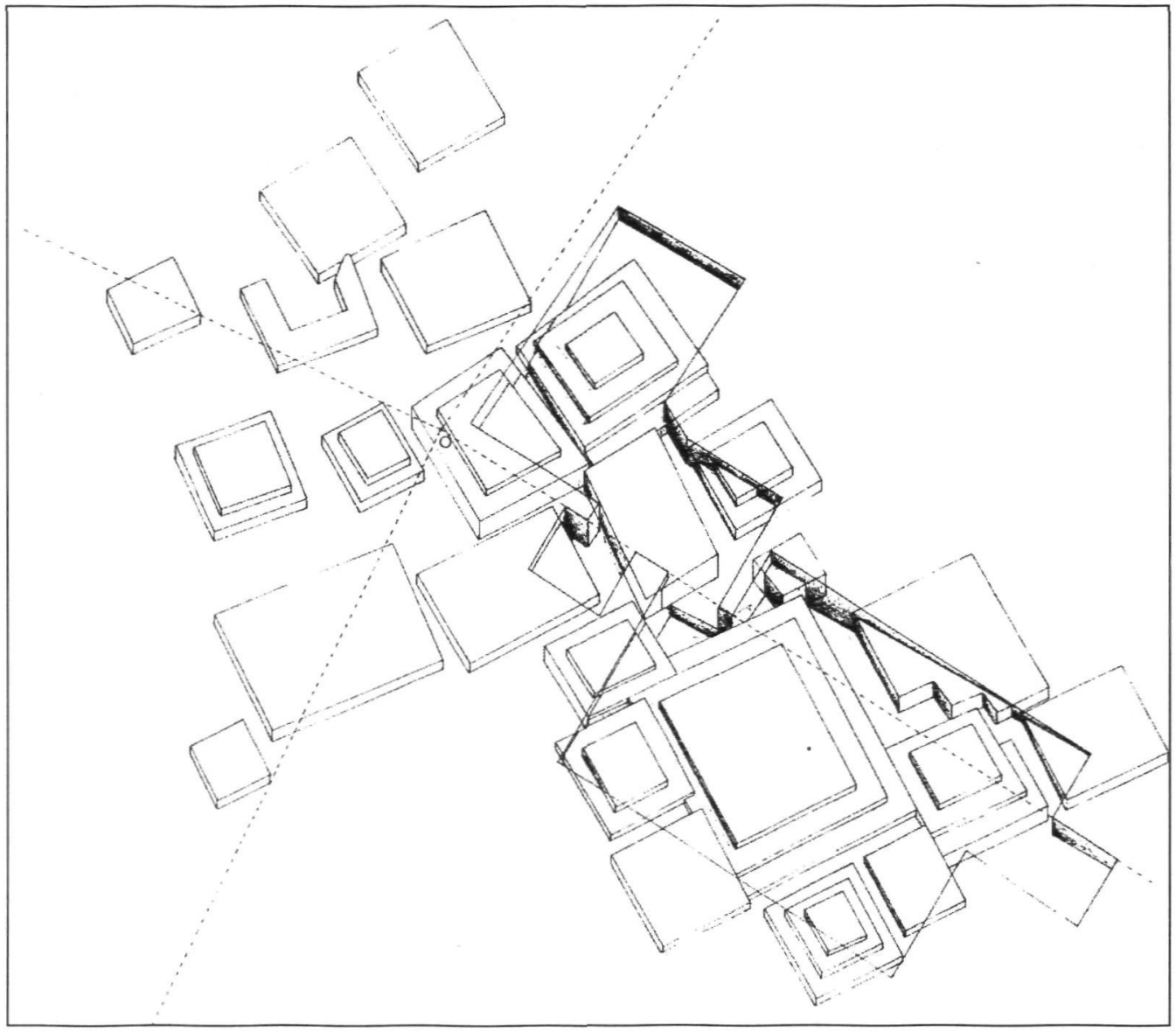

Figura 1. Axonometría de la necrópolis tumular de Los Villares. Campaña de 1987. 
semitismo-panhelenismo" ha rodeado líneas de investigación acentuadas, quizás, por las propias características del sur peninsular, una de las mejores zonas del Mediterráneo en donde se documenta el impacto colonial fenicio en sus fases más arcaicas (Aubet Semmler, 1986, 612). Así, las publicaciones de las Actas de Colonia y Roma, ambas en 1979; o las Jornadas sobre Colonizaciones Orientales, celebradas en Huelva al año siguiente, son un buen reflejo de este interés. Tanto es así que las citadas publicaciones dieron lugar a la acuñación del término "año de los fenicios" (Aubet Semmler, 1980-81, 263) que, si bien es anecdótico, no era sino reflejo de una realidad. Con posterioridad los trabajos de conjunto de Aubet y Del Olmo Lete cerraban, bibliográficamente, todo una época de investigación arqueológica iniciada allá por los años sesenta (Del Olmo Lete y Aubet Semmler, 1986, 5). Dicha labor quedó justamente reconocida en la pasada exposición internacional I Fenici, celebrada en Venecia (Moscati, 1988, 22).

Paralelamente, en estos últimos años el desarrollo de los trabajos arquoelógicos llevados a cabo en el sur peninsular y, más concretamente, en Huelva están sacando a la luz nuevos materiales que obligan a un replanteamiento general del problema, ya planteado en los años 40 . Nos referimos al debatido problema del reparto de mercados -áreas de distribución entre griegos y fenicios en la Península Ibérica (F. Pacheco, 1948). Analizado, en su momento, desde una perspectiva demasiado simple se excluían agentes y materiales como, por ejemplo,el etrusco que hoy tiene carta de naturaleza en la investigación arqueológica española. Así, el reciente congreso celebrado en Barcelona sobre el impacto Etrusco en la Península Ibérica es revelador. Parece necesario, pues, empezar a valorar novedosos materiales de clara procedencia etrusca que colaboraron con peso en la difusión de ideas, tan importantes, como los ritos del vino y del symposium. Apuntado como línea de trabajo por algunos investigadores (Olmos Romera, 1987) hoy contamos con documentación arqueológica (Blánquez Pérez, 1990).

La aparición de cerámicas griegas en distintos solares de la ciudad de Huelva: Plaza de la Pitillera, calle del Puerto n. 6 y 9 y calle de la Botica n. 10 y 12 (Fernández Jurado, 1985, 9) ha venido a demostrar la presencia directa de agentes griegos desde finales del s. VIII. Los materiales arqueológicos abarcan hasta finales del s. VI a.C., con especial abundancia en la primera mitad de este último siglo. Presentados, por primera vez, en 1982 por su excavador (Garrido Roíz, 1983, 555), luego han sido objeto de diferentes estudios proponiendose distintas interpretaciones (Olmos y Cabrera, 1980; Olmos y Garrido Roíz, 1982, 243-264; Olmos, 1982, 393406). Posteriores trabajos arqueológicos de especialistas ratifican su importancia (Fernández Jurado, 1984; revisado en Cabrera, 1986, 575-583; recientemente Fernández Jurado, 1989).

La evidencia arqueológica que estas cerámicas implican -la presencia de un comercio foceo (Fernández Jurado, 1986, 572 y Cabrera, 1986, 581)-completa y fortalece tesis científicas aportadas en estudios paralelos por otros investigadores. Para éstos el factor griego, en los siglos VI y $\mathrm{V}$ a. de C., sería imprescindible a la hora de explicar la formación de la cultura ibérica; y, muy especialmente,en el sureste de la Meseta.

Trabajos publicados en los últimos años documentan la existencia de auténticas rutas de comunicación y comercio por las que la cultura griega, por mano focea-ampuritana, actuó como revulsivo en las poblaciones autóctonas preexistentes. Planteado en un primer momento como hipótesis, con gran intuición (Maluquer de Motes, 1985); posteriores trabajos lo han matizado con materiales (Domínguez Monedero, 1988) y demostrando mediante excavaciones (Blánquez Pérez, 1990, a y b). El resultado final fue, como hemos apuntado, la configuración definitiva de los pueblos ibéricos del levante y sureste peninsular (Maluquer de Motes, 1981, 211; Chapa Brunet, 1982; Muñoz Amilibia, 1984). Ello no supone la negación de unas más antiguas 


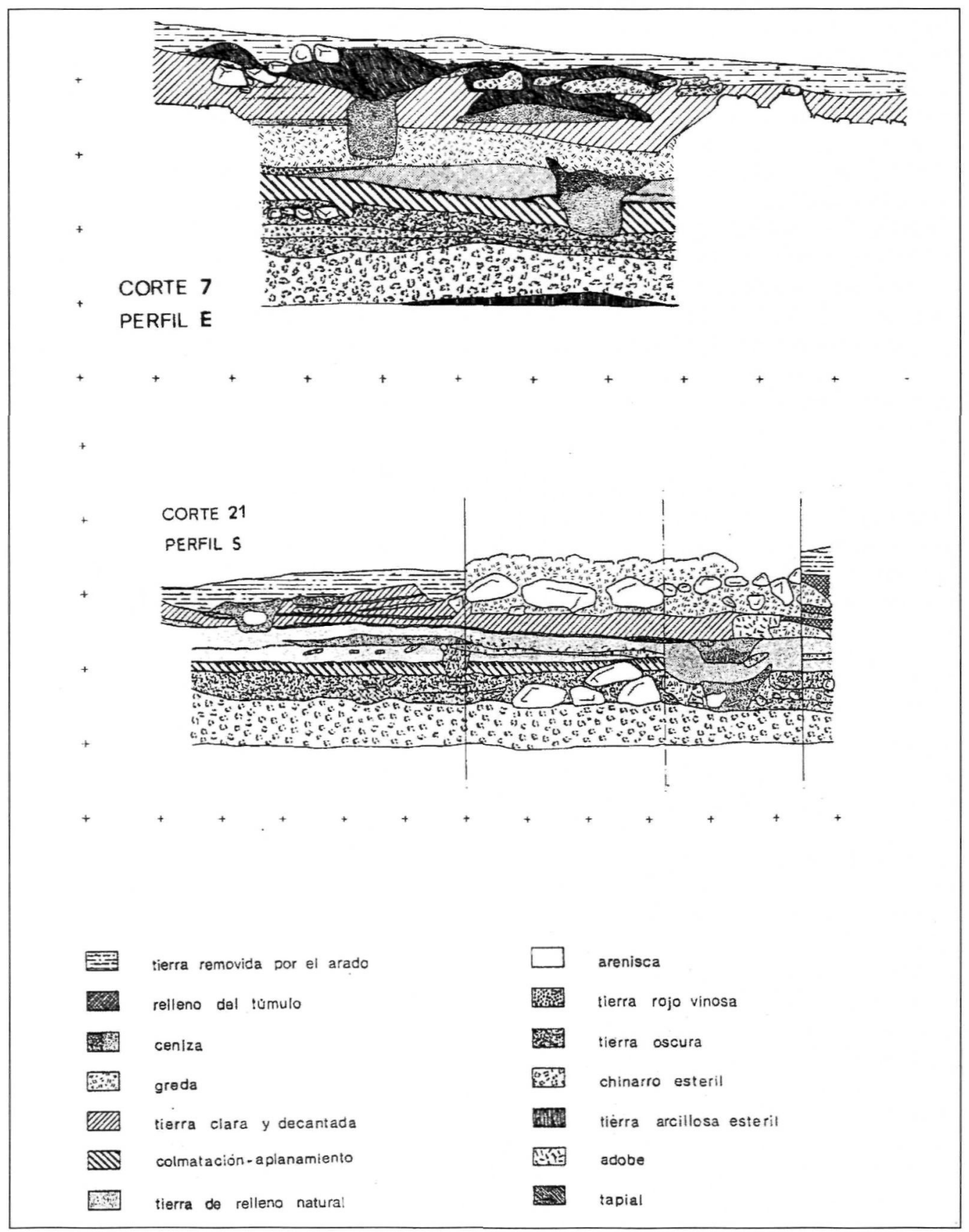

Figura 2. Estratigrafías arqueológicas. A) Corte 7, perfil E. B) Corte 21, perfil S. C) Leyenda. En todas ellas se documentan las tres fases de la necrópolis. 


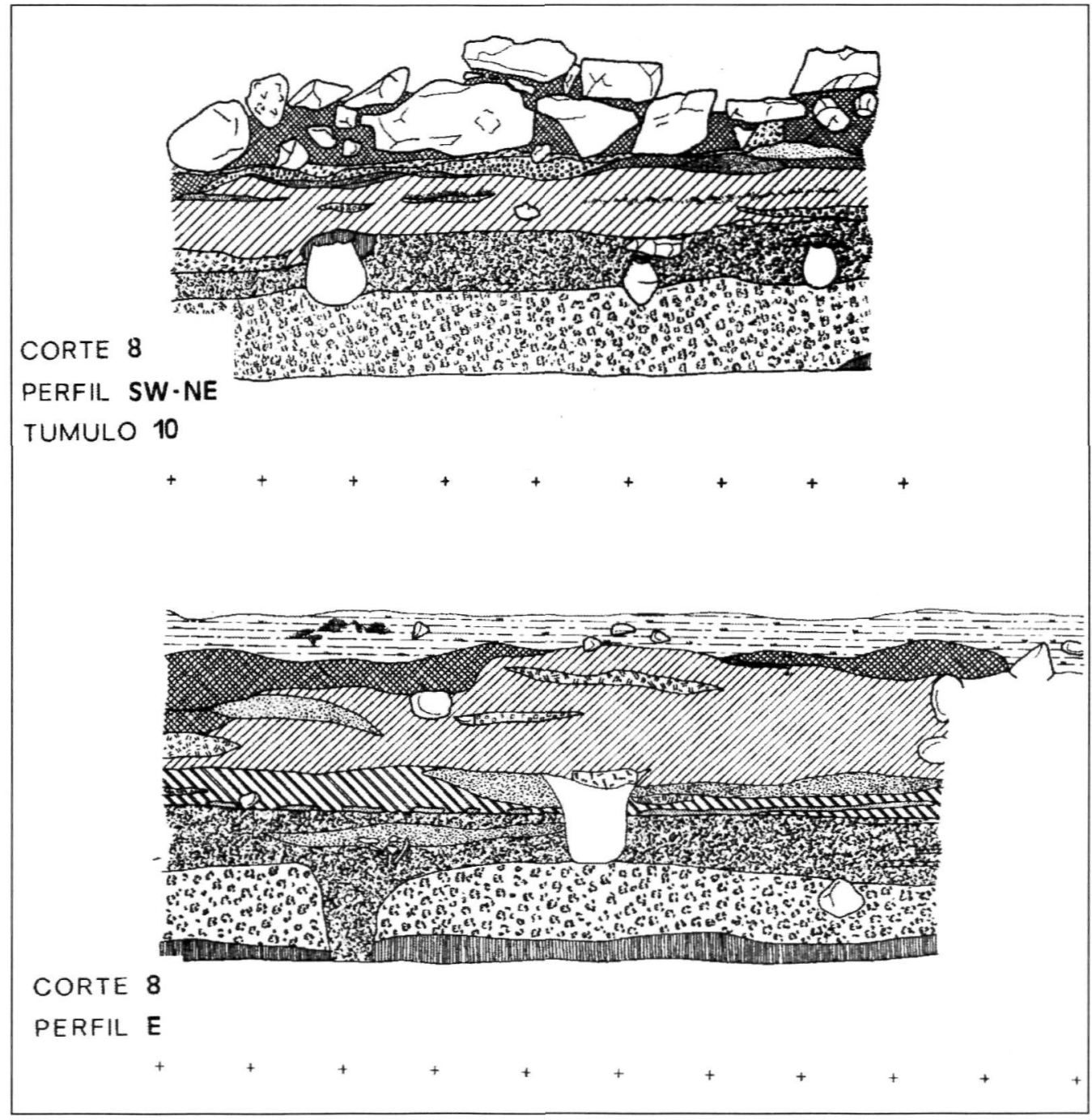

Figura 3. Estratigrafía de la cuadrícula 8: perfil SW-NE, con el Túmulo. 10 y perfil E.

influencias orientalizantes, importantísimas e imprescindibles. Pero, parece ser, no del todo suficientes para materializar, en todas sus facetas, la cultura ibérica. Faltos, en sí mismas, para actuar como revulsivo definitivo (Blánquez Pérez, 1984, 199 y 1990 a).

Queda posibilitado, pues, hablar de la presencia griega en el sureste peninsular y Levante, con una penetración deliberada hacia el interior de la Meseta (Domínguez Monedero, 1986 y Blánquez Pérez, 1986-87).

A tenor de las excavaciones en necrópolis ibéricas como Cabezo Lucero (Guardamar del Segura, Alicante); Camino de la Cruz y Los Villares (ambas en hoya Gonzalo, Albacete); La 
Boadilla (Alcaudete, Jaén); Estacar de Robarinas (Cástulo-Linares, Jaén) y del palacio de Cancho Roano (Zalamea de la Serena, Badajoz) se abren nuevas perspectivas en al investigación. Es conveniente valorar, de nuevo, el "factor griego" en la colonización de la Península Ibérica y, por ende, del proceso formativo de las culturas ibéricas en la meseta sur oriental.

La importancia de los nuevos descubrimientos, al margen de su valor material, reside en el ser acicate de investigaciones colaterales. Por un lado, la posibilidad de revisar, a la luz de los nuevos datos, excavaciones antiguas (Blánquez Pérez, 1986); por otro, por la relectura de las fuentes (Morel, 1984, 481), ni mucho menos agotadas en sus posibilidades interpretativas (Domínguez Monedero, 1985, 376).

Centrándonos ya en la zona geográfica que nos ocupa, el sureste de la Meseta, destacamos en primer lugar la necrópolis de enterramientos tumulares de Los Villares, en Hoya Gonzalo, Albacete (Blánquez Pérez, 1984, 1988 b y 1990 a) (ver lám. 1). Los materiales de importación que formaban parte de los ajuares funerarios testimonian la existencia de un comercio con el mundo exterior mediterráneo. En efecto, aryballoi de fayenza; lekythoi de figuras rojas; kantharoi de SaintValentin, abundantes piezas de barniz negro y un importantísimo conjunto escultórico y arquitectónico, sustentados todos en una sólida estratigrafía arqueológica (Blánquez Pérez, 1990 a) (ver Fig. 2 y 3), plantean dos cuestiones básicas de análisis.

a) Por un lado, definir el tipo de comercio que se estructura a tenor del material documentado en la necrópolis. Determinar si se trata de un caso aislado o si, por el contrario, vemos una repetición sistemática en otros yacimientos. En caso afirmativo sería necesario establecer posibles interrelaciones, etc.

b)Una vez custionados los aspectos anteriores es necesario determinar qué gentes-centros serían capaces de satisfacer una demanda de semejantes características.

Y todo ello deberíamos encuadrarlo entre el tercer cuarto del s. VI a.C. y los inicios del s. IV a. de C; fechas éstas en que encuadramos los materiales y estratigrafías de Los Villares (Blánquez Pérez, 1988 a, 349; 1988 b, 37 y desarrollada en Blánquez Pérez 1990a). Sólo así podremos solucionar problemas arrastrados de antiguo, así como entender la procedencia de materiales griegos en la Península (Domínguez Monedero, 1986, 602).

Los elementos de importación documentados en la necrópolis de Los Villares no se presentan como objetos aislados, hecho éste que reflejaría una comercialización casual. Su repetición y coherencia tipológica evidencia, bajo nuestro punto de vista, un comercio de producciones seriadas sólo concebible en un circuito estructurado.

Su aumento cuantitativo, manteniendo las pautas de uniformidad e interrelación tipológica, pone de manifiesto el hecho de que estamos asistiendo al afianzamiento de un comercio bien definido que implica, además, un más que correcto conocimiento del medio geográfico por parte de los agentes comeciales. En este sentido, la aparición de un segundo silicernium en la pasada campaña de excavaciones (1989), esta vez dentro de una tumba con cubrición tumular, corrobora arqueológicamente lo hasta aquí postulado (ver Lám. 2).

Los materiales aparecidos son, en su mayor parte, piezas de importación. Pero creemos todavía mas importante el hecho de que coincidan, uno y otro silicernium, en los tipos de materiales: barniz negro ático; pasta vítrea, joyería áurea y marfiles de clara procedencia etrusca .

Se han reconstruido más de una docena de bolsales, con y sin decoración estampillada; tres lekythoi; tres skyphoi; tres páteras; un plato; dos askoi; dos aryballoi y un amphorisco de pasta vítrea. Todos ellos componen, entre otras piezas, el ajuar del primer silicernium. El segundo, excavado en la campaña del 1989 y todavía en proceso de restauración presenta también una 
mayoritaria presencia de cerámicas griegas entre las que destacamos varios kantharoi completos del tipo St. Valentin. En ambos ejemplos la funcionalidad de las piezas, así como su ubicación en necrópolis, pone de manifiesto la existencia de ritos funerarios en los que perfumes y vino fueron los protagonistas. Es decir, libación y symposium.

Si piezas y actos formales coinciden con lo conocido en Grecia creemos correcto suponer aquí, en la Península Ibérica, la aceptación también de las ideas y mitos originales, aun con evidentes grados de aceptación-readaptación. Todo ello asumido y asentado en la sociedad ibérica (ver Fig. 1).

$\mathrm{Si}$, paralelamente, realizamos una rápida revisión de los materiales documentados en otras excavaciones (Blánquez Pérez, 1986, 1988 a y 1990 a) vemos que aparecen los mismos tipos. Aryballoi de fayenza los encontramos en el Molar (San Fulgencio, Alicante); Hoya de Santa Ana (Chinchilla, Albacete); La Bobadilla (Alcaudete, Jaén) y Cancho Roano (Zalamea de la Serena, Badajoz) (Shefton, 1982, 359).

Todos estos ejemplos de Naucratis presentan una coincidencia tipológica y decorativa: se tratan de aryballoi y sus galbos presentan un acabado reticulado, o gallonado(ver Fig. 4 y Lám. 3 a). Pero más interesante es, todavía, el espacio geográfico que definen: una vía de penetración hacia el interior ratificada por la presencia de otros materiales. Serían las citadas cerámicas de Saint-Valentin; las Cástulo-Cup; o las piezas de marfil y/o hueso decoradas con motivos geométricos y figurados, de posible procedencia etrusca (Fig. 5 b ).

Si establecemos una ruta lineal entre los yacimientos con cerámicas de St. Valentin marcamos, de nuevo, el itinerario Alicante-Albacete-Jaén-Badajoz, coincidente con el visto para la fayenza. La Bastida (Mogente) y Covalta (Albaida) en Valencia; El Puntal (Salinas), en Alicante; Archena, en Murcia; Villares y Hoya de Santa Ana, en Albacete; Cástulo en Jaén y el propio Cancho Roano, en Badajoz, no dejan lagunas que sean significativas (Maluquer de Motes, 1974, 435 y ss.).

Sólo, por último, queremos añadir los hallazgos de Cabezo del Tío Pío, publicados de antiguo (Beazley, 1943, 47), aunque sin clasificar. Nosotros los encuadramos dentro de los tipos IIIIV de Howard y Johnson (Howard y Johnson, 1954, 192 y ss.). Estos, junto a los vistos en Puig dels Molins, corresponden a ejemplares de alta cronología. Es, pués, muy sugerente el que algunos de los tipos más antiguos se documenten en el sur peninsular e isla de Ibiza.

Analicemos, a continuación, los agentes capaces de comercializar los citados materiales griegos, estableciendo contacto directo con las culturas autóctonas, así como de mantener una continuidad en el tiempo.

La repetición tipológica, arqueológicamente demostrable, contradice un comercio de intermediarios. De manera paralela, la presencia de un comercio foceo en la península, a raíz de las investigaciones en la zona de Huelva, cobra consistencia (Olmos Romera, 1982, 39). El relativo decaimiento del comercio del estaño, unido a la apertura de nuevas rutas noreuropeas, por el Ródano, en las que el mundo focense no estuvo ajeno; o el posible agotamiento de las minas de plata del suroeste peninsular (Garrido Roíz, 1982, 416) debido, seguramente, a limitaciones técnicas (Fernández Jurado, 1984, 55) debieron provocar un decaimiento general del suroeste peninsular.

Ello debió provocar una doble respuesta. Por una parte, el cese brusco de las importaciones griegas, incapaces las poblaciones indígenas de acceder a este comercio de lujo (Cabrera, 1986, 581); y, por otro, la búsqueda de nuevas zonas de explotación.

Todo ello supuso, bajo nuestro punto de vista, una revalorización del sureste, levante, alta Andalucía y sureste meseteño. Esta última, contigua a todas, cumplía una indudable función de 
puente que la convirtió, en cierto modo, en un área privilegiada con personalidad propia, mucho mas que un mero cruce de caminos.

Así se explica el fuerte poso orientalizante y la pervivencia de elementos de la cultura tartésica; la existencia de materiales griegos, desde finales del s. VI a.C., sin interrupciones y en aumento a partir de entonces... Características, todas ellas, del arranque de la cultura ibérica en el Sureste de la Meseta.

Emporion debió ser, con seguridad, el punto fundamental en la redistribución de la gran mayoría de los elementos griegos en la península. Desde los materiales que fechamos con mayor antiguëdad, con una cronología del tercer y cuarto cuarto del s. VI a.C. -con las cerámicas de fayenza- (Ripoll, Sanmartí, 1978, 34); hasta las cerámicas griegas de figuras rojas -las oinochoes (Forma III) de Ullastret y Los Villares-; la cerámica de Saint-Valentin, de finales del s. V a.C.; y también ¿por qué no? redistribuidora de materiales de procedencia etrusca.

Cabe destacar en esta última línea la existencia de pequeños cofres decorados con placas de marfil. Si bien parece no constituyeron mercancía propia sino, más bien, bienes de prestigio (Jannot, 1986, 409) los encontramos formando parte de ajuares funerarios ibéricos. Los últimos hallazgos de Los Villares (Campañas de 1983 y 1989), así como de otros similares aparecidos con anterioridad que podemos reinterpretarlos con este nuevo sentido: Ampurias, Hoya de Santa Ana, quizás Cancho Roano, obligan a una hipótesis en cuanto a líneas de distribución y agentes comerciales. Y es en este sentido donde, de nuevo, encontramos el protagonismo de Emporion.

Es sabido que, durante el siglo VI, Emporion estuvo bajo la órbita massaliota. Lo vemos, claramente, en sus primeras acuñaciones con el cuadro incuso anepigráfico; en su estrato IX encontramos materiales jonio-focenses, corintios y áticos. Asimismo, ya para entonces, habría exploraciones-tanteos por la costa levantina y sureste de la meseta. Su reflejo sería la cerámica de fayenza.

En el siglo V, favorecida por el temporal decaimiento de Massalia, Emporion adquirió un rápido y progresivo fortalecimiento gozando de plena independencia comercial. Dicha autonomía le permitió comerciar, directamente, con la propia Atenas, sobre todo a partir del 480 (Picazo, 1980, 156). Las importaciones que a partir de entonces se documentan en la costa levantina sólo Emporion habría podido adquirirlas y si todavía hoy son escasas se debe, probablemente, a falta de investigación. De hecho, los trabajos arqueológicos realizados en los últimos años son prueba de ello. Valgan como ejemplo las excavaciones en el Puig de Benicarló (Sanmartí y Gusi, 1876; Gusi y Oliver, 1987, 106 y 124 especialmente); o, en Cabezo Lucero (Aranegui, C. et alii, 1986).

Uno de los mas claros ejemplos del protagonismo de Emporion se materializa tras los descubrimientos cerámicos de Saint-Valentin en las tierras del sureste meseteño (ver Lám. 3 b). Tanto las aparecidas en la necrópolis tumular de Los Villares; como aquellas con decoración de guirnaldas, tras la revisión- restauración hecha en la Hoya de Santa Ana (Amitrano, 1985).

El que la intermediaria exclusiva de este material fuera Emporion no debe extrañarnos. Constituye la prueba material de las relaciones de este enclave con la propia Atenas. Fue, pués, una mecánica paralelizable a la del coral rojo (Picazo, 1977, 27 y ss.); o aquellas otras de pintura "en relieve" propias de producciones griegas de finales del s. V a.C. y que también encontramos en el interior de la meseta.

La cuantificación y ordenación cronológica del material de Saint Valentin da una proporción muy parecida entre formas antiguas y tardías. Ello denota un comercio continuado, no de tanteos previos, de una actividad comercial con más de un siglo de duración. Los ejemplos son claros: 


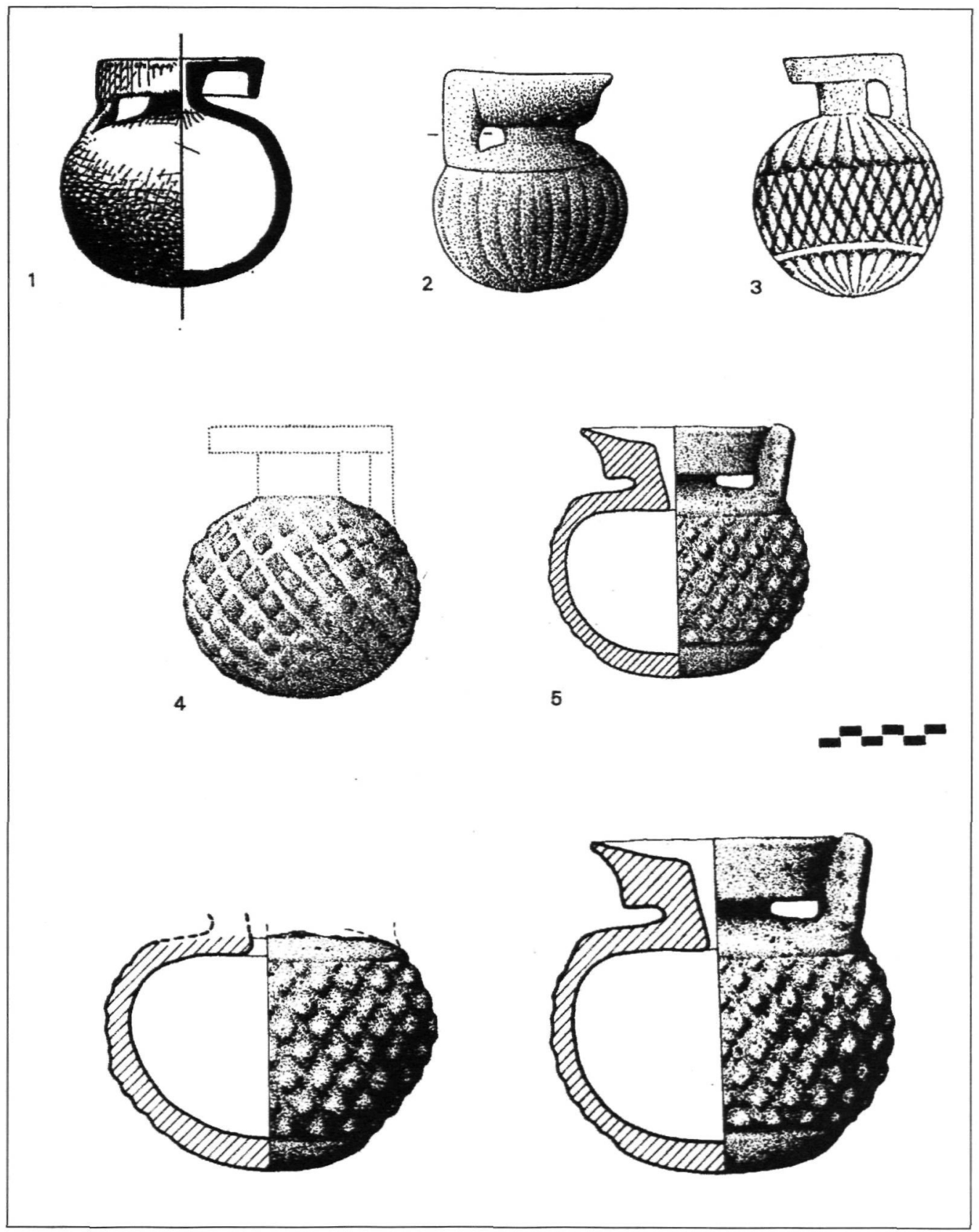

Figura 4. Aryballoi de fayenza: 1 Necrópolis de Ampurias (según M. Almagro); 2 Hoya de Santa Ana; 3 y 4 Cancho Roano (según Maluquer de Motes); 5 Los Villares. B) Aryballoi de Los Villares (E: 1/1) de la Tumba 22 b. 


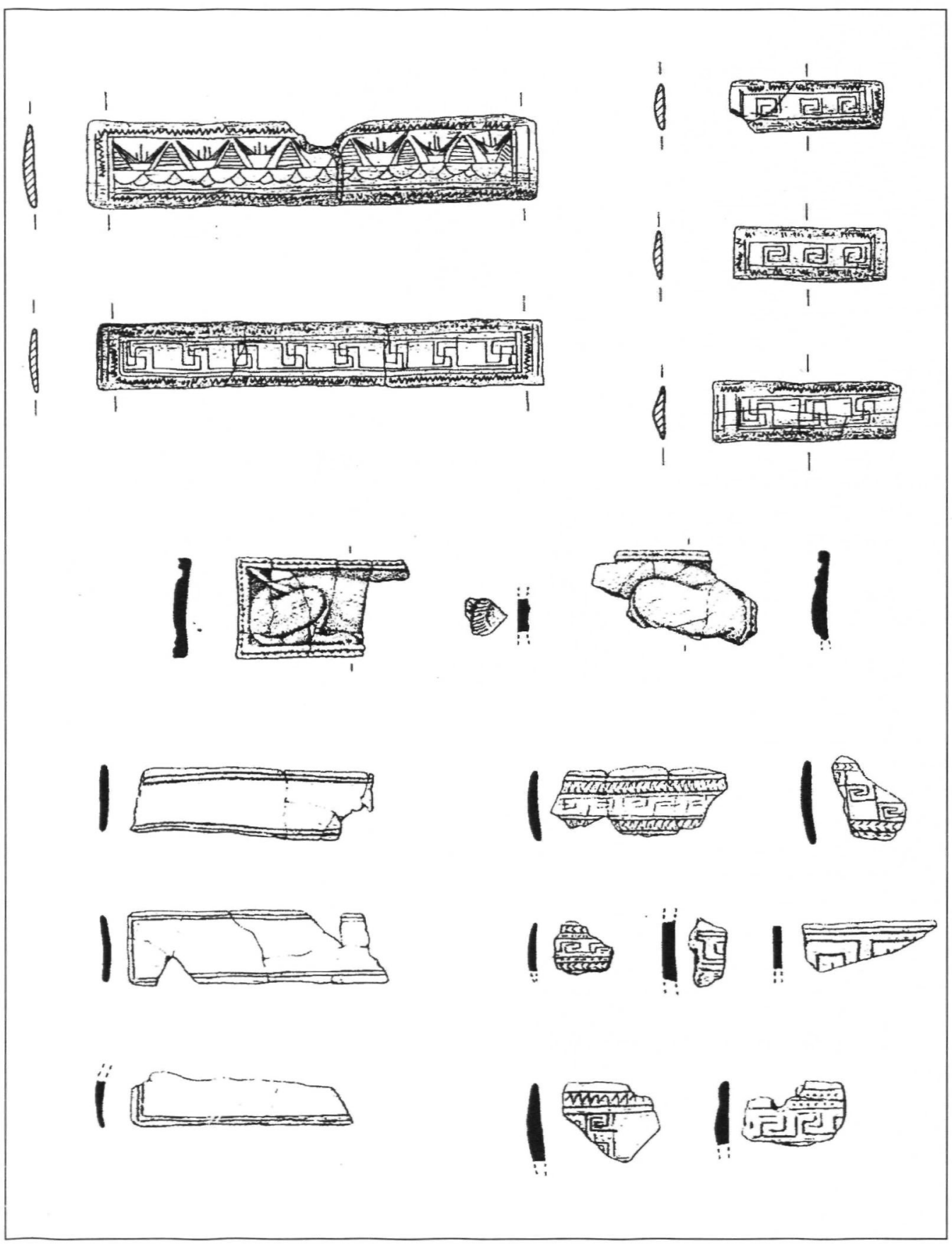

Figura 5. Placas trabajadas: A) Hoya de Santa Ana; B) Los Villares (silicernium de 1983). 
fragmentos del tipo VII en Los Villares (silicernium del 1983 y del 1989); el skyphos de la Hoya de Santa Ana, con paralelos claros tras la limpieza de sus falsificaciones, en Cancho Roano; o, por último, el que aparezcan en contextos materiales muy parecidos: placas de hueso y/o marfil, no de procedencia griega (Maluquer de Motes, 1983, 41 y 102), sino posiblemente etrusca (Martelli, 1985).

Lo que observamos es una aceleración, sin precedentes, en la importación de cerámicas griegas a partir del último cuarto del siglo $\mathrm{V}$ a. de $\mathrm{C}$. Pero, tanto el punto de redistribución (Emporio), como la red de caminos (por las rutas del interior), no experimentaron cambios substanciales.

Es por todo lo expuesto que el sustrato indígena preexistente, aquel sobre el que se había asentado el mundo orientalizante, la herencia tartésica, incluso elementos hallstáticos a través de la Meseta norte, experimentó en su seno una última y definitiva transformación. Con la llegada del mundo griego-foceo finalizaría un proceso de maduración cultural cuyo resultado fue la configuración definitiva de la Cultura Ibérica en el sureste de la Meseta. Dicho proceso, a finales del s. VI a. de C. ya estaría culminado.

Quedaría definido, pues, un binomio arqueológicamente demostrable para nuestra zona de estudio: configuración definitiva de la cultura ibérica-fenómeno comercial griego-foceo. La búsqueda de nuevas fuentes minerales; posiblemente también de sal y esparto (Domínguez Monedero, 1986, 609), en unión a un activo comercio foceo provocaron una reacción interna en las poblaciones autóctonas. Las cerámicas de fayenza, importaciones etruscas, cerámicas áticas, de Saint-Valentin, los enterramientos con cubrición tumulares, o la propia escultura en piedra son prueba de ello (Blánquez Pérez, 1990 a).

Los materiales aquí reseñados constituyen, en sí mismos, algunas de las evidencias materiales de esta nueva identidad y testimonian, de modo paralelo, la aceptación de ritos como la libación, el perfume, el symposium, o el enterramiento tumular acordes con el sentir griego. Como es lógico, todos ellos con un suficiente grado de transformación-reinterpretación que no hacen sino poner de manifiesto la propia personalidad de aquellas gentes. Así, pues, hablar de Cultura Ibérica en el Sureste de la Meseta equivaldría a resaltar el término de helenización. 

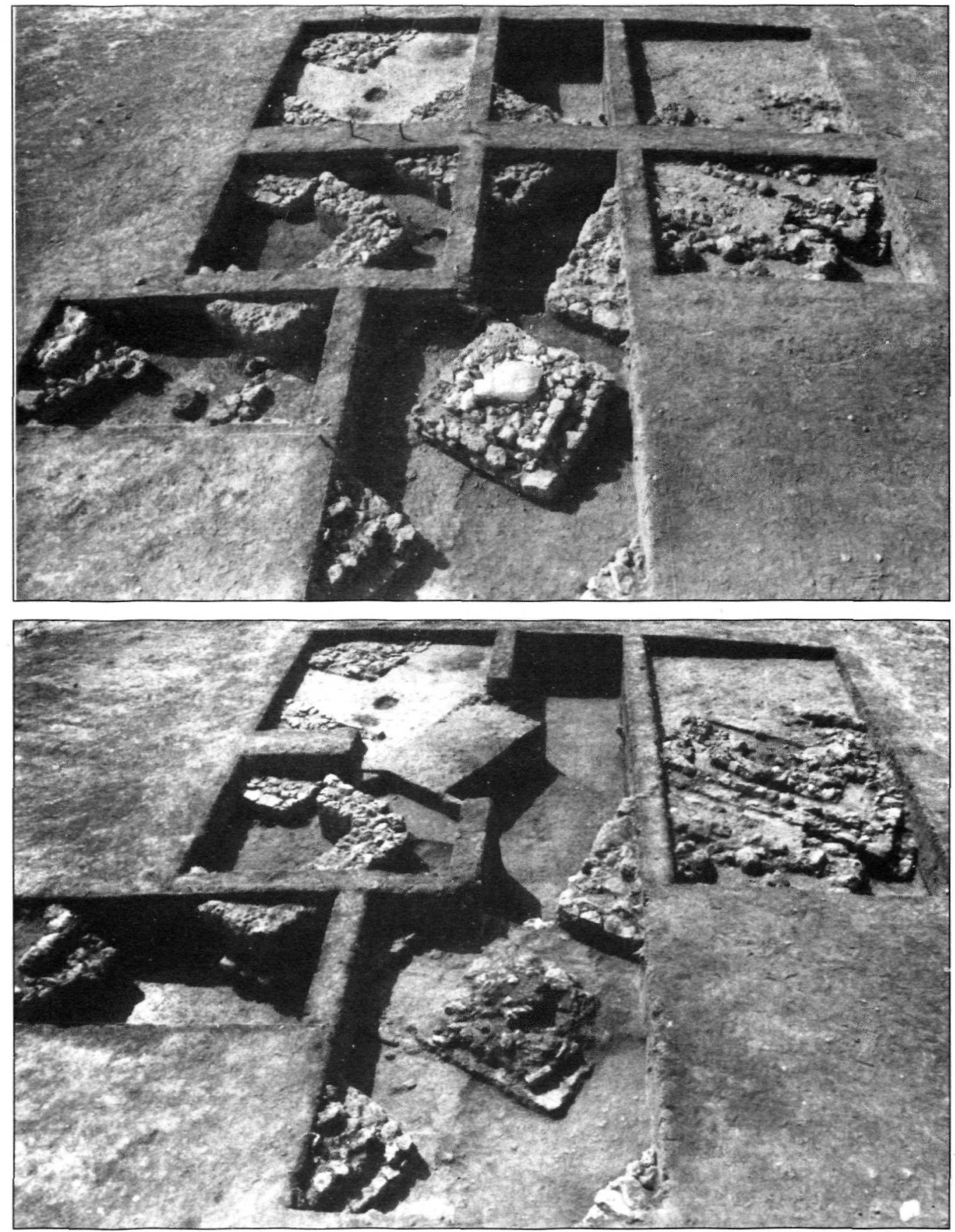

Lámina 1: A) Vista general de la excavación inicio campaña del 84. B) Finalización de la campaña del 84. 

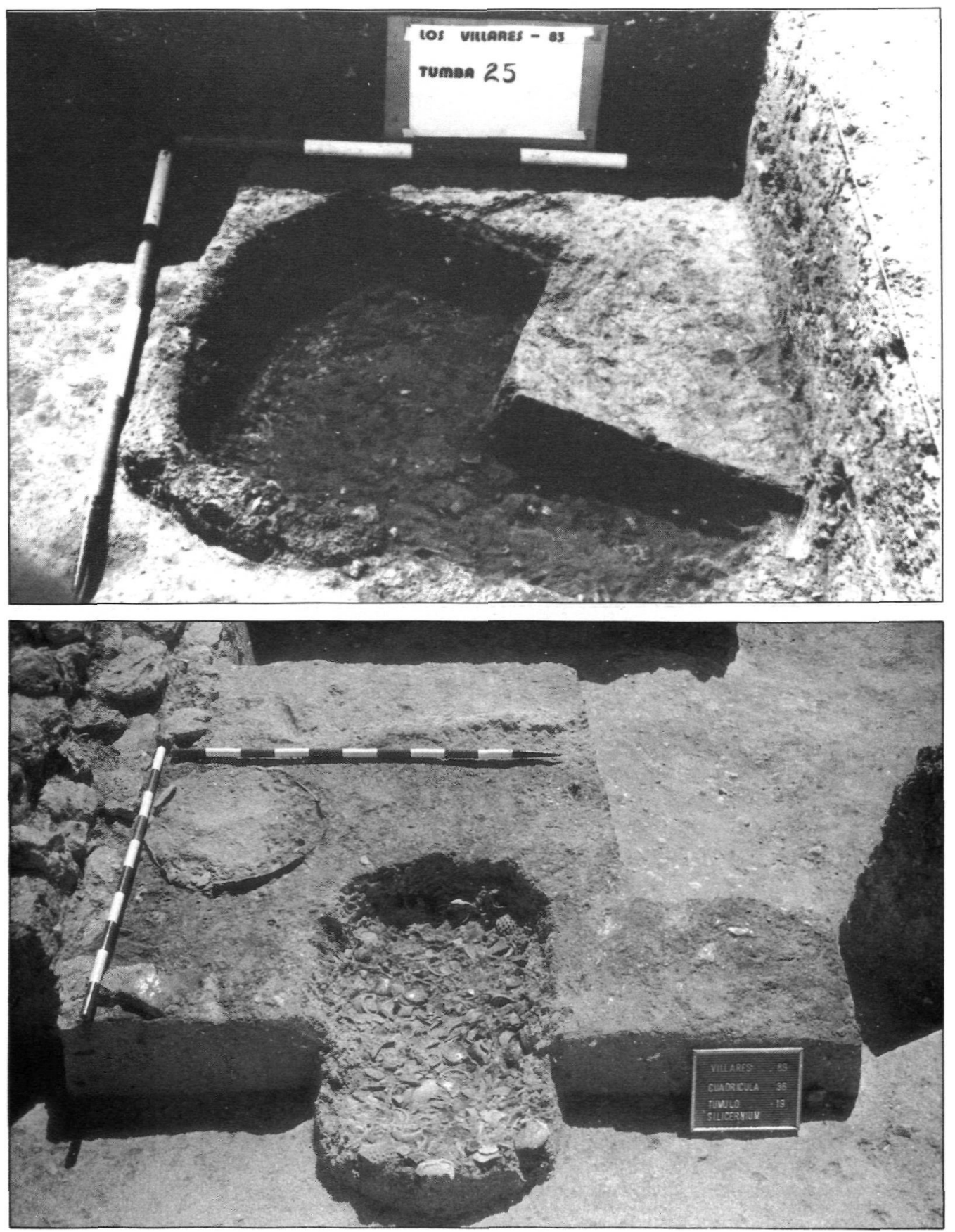

Lámina 2: Los Villares: A) silicernium 1983; B) silicernium 1989. 

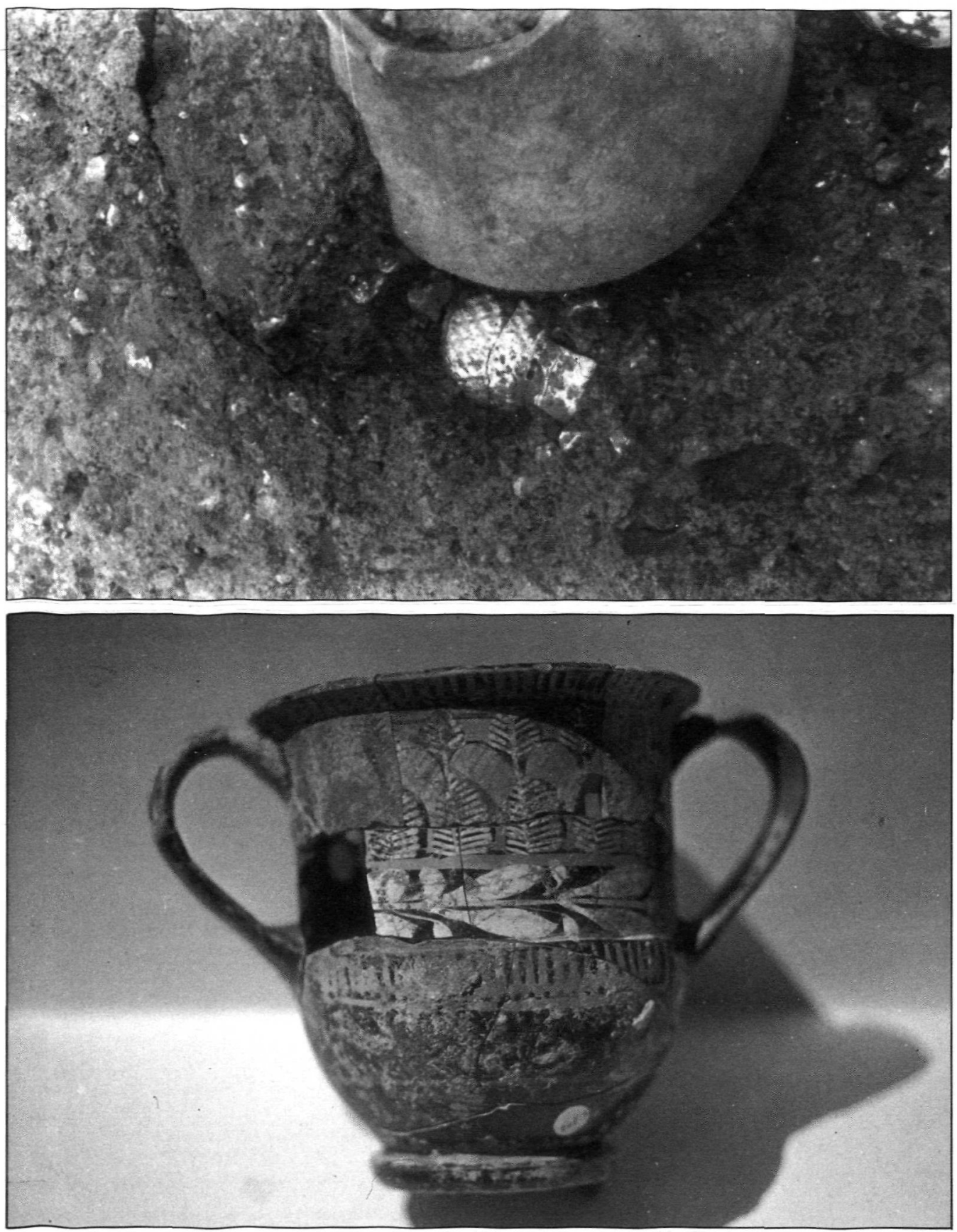

Lámina 3: Los Villares: A) Tumba 22 b (Fase I) con dos aryballoi como parte de su ajuar funerario. Finales del s. VI a.C. B) Kantharos de St. Valentin del silicernium de 1989. 


\section{BIBLIOGRAFIA}

AMITRANO BRUNO, R.F., 1988: "Un replanteamiento de la restauración de algunas piezas de barniz negro de Hoya de Santa Ana, Albacete”. I Congreso de Historia de Castilla-La Mancha. Ciudad Real, 1985, Vol. III, 317-326.

Aranegui, C., Jodin, A.; Llobregat, E; Rouillard P. y Uroz, J., 1986: "Fouilles du site ibèrique de Cabezo Lucero (Guardamar del Segura-Alicante). Cinquième Campagne, 1983”. Mélanges de la Casa Velazquez, Tomo XXII. 549-558.

Aubet Semmler, M. A., 1980-81: "Nuevo impulso internacional a los estudios de la colonización fenicia". Mainake, II-III, 263-266.

Blanquez Perez, J., 1984: "Las necrópolis ibéricas de la provincia de Albacete". Congreso de Historia de Albacete, Vol. I, Arqueología y Prehistoria, Alabacete, 185-209.

... 1986-1987: "Notas acerca de una revisión de la necrópolis ibérica de La Hoya de Santa Ana (Chinchilla). Albacete”. Homenaje al Prof. Dr. Gratiniano Nieto Gallo, Cuadernos de Prehistoria y Arqueologia, 13-14, Vol. II, 9-27.

... 1988 a: "La segunda campaña de excavaciones en la necrópolis ibérica de Los Villares, en Hoya Gonzalo, Albacte. Estado de la cuestión". I congreso de Historia de Castilla-La Mancha. Ciudad Real, 1985, Vol. II, 345-357.

... 1988 b: "Los enterramientos de estructura tumular en el mundo ibérico". I Congreso Peninsular de Historia Antigua. Santiago de Compostela, Vol. II, 5-38.

... 1990 a: La formación del mundo ibérico en el Sureste de la Meseta. (Estudio arqueológico de las necrópolis ibéricas de la provincia de Albacete). Instituto de Estudios Albacetenses. Albacete.

... 1990 b: "La Vía Heraklea y el Camino de Aníbal. Nuevas interpretaciones de su trazado en las tierras del interior”. Simposio. La Red Viaria en la Hispania Romana. Zaragoza, 65-76.

Cabrera Bonet, P., 1986: "Los griegos en Huelva: Los materiales griegos". Homenaje a Luis Siret (19341984). Cuevas de Almanzora, Sevilla, 575-583.

Chapa Brunet, T. 1982: "Influences de la colonisation Phocéenne sur la sculture Ibérique". Parola del Passato. Revista di Studi Antichi, XXXVII, 374-392.

Del Olmo Lete, G. Y Aubet Semler, M. E., 1986: Los fenicios en la Peninsula Ibérica, Barcelona, I y II.

Dominguez Monedero, A., 1985, "Focea y sus colonias: a propósito de un reciente coloquio". Gerion, 3, 357-377.

... 1986, "Reinterpretación de los testimonios acerca de la presencia griega en el Sudeste Peninsular y Levante en época arcaica”. Homenaje a Luis Siret (1934-1984). Cuevas de Almanzora, Sevilla, 601612.

... 1988: "Algunas observaciones en torno al comercio continental griego en la Meseta meridional". I Congreso de Historia de Castilla-La Mancha, Yol. III, 327-334.

FERnANDEZ JuRADO, J., 1984: "La presencia arcaica en Huelva". Monografias arqueológicas. Colección Excavaciones en Huelva 1.

... 1986: "Fenicios y Griegos en Huelva". Homenaje a Luis Siret (1934-1984). Cuevas de Almanzora, Sevilla, 562-574.

... 1989: "La orientalización en Huelva". Tartessos. Arqueología Protohistórica del Bajo Guadalquivir. Barcelona, 339-373.

Figueras Pacheco, F., 1947: "Griegos y púnicos en el Sudeste de España. Proceso geográfico-histórico de la colonización”. Crónica del III Congreso Arqueológico del Sudeste, Madrid, 187-201. 
Garrido RoIZ, J. P., 1983: “Avance sobre las excavaciones de urgencia de la calle del Puerto. Huelva”. XVI Congreso Nacional de Arqueología. Murcia-Cartagena, 1982. Zaragoza, 549-559.

GUSI, J. Y OLIVER, A., 1987: “La problemática de la iberización en Castellón”. Actas de las Jornadas sobre mundo ibérico. Jaén, 1985, 49-136.

Howard, S. y Johnson, P., 1985: “The Saint-Valentin vases”. American Journal of Archaeology. Vol. 58, n. 3, 191-297.

JANNOT, J. R., 1986: "Sur les coffrets archaïques étrusques à décoration d'ivoire et d'os: hypothèses sur la production et la diffusion". Italian Iron Age Artifacts. Papers of the sixth British Museum Classical Colloquium. London, 1982, 405-415.

Lopez Domenech, R., 1988: "Los vasos áticos del s. IV a.C., elemento de interacción comercial en la región de Albacete". Congreso de Historia de Albacete. Vol. I. Arqueología y Prehistoria, Albacete, 139-143.

Maluquer de Motes, J., 1974: “Cerámica de Saint-Valentin en Ullastret (Gerona)”. Miscelánea Arqueológica. XXV Aniversario de los Cursos de Ampurias. Barcelona, Vol. I, 411-437.

... 1981: "El peso del mundo griego en el arte ibérico". La Baja época de la cultura ibérica. Actas de la Mesa Redonda. Madrid, 302-216.

... 1985: "Comercio continental focense en la Extremadura Central". Ceràmiques grecques $i$ helenístiques a la Peninsula Ibérica. Empúries, 19-25.

MARTElli M., 1985: "Gli avori tardo-arcaici: bottegue e aree di difusione". Il comercio etrusco arcaico. Quaderni del Centro di Sudio per L'Archelogia Etrusco-Italica, 9, 1983, 207-248.

Morel, J.P., 1982: "Les Phocéens d'Occident: nouvelles donnés, nouvelles approches”. I Focei dall'Anatolia all Oceano.Parola del Passato. Rivista di Studi Antichi. Vol. XXXVII, 479-500.

MosCati, S., 1988: I Fenici. Milano.

Muñoz Amilibia, A.M., 1983: "La plástica ibérica en Albacete". Congreso de Historia de Albacete. Vol. I Arqueología y Prehistoria. Albacete, 145-156.

Olmos Romera, R., 1982: "La cerámica griega en el Sur de la Península Ibérica. La aportación de Huelva". Parola del Passato. Rivista di Studi Antichi. Vol. XXXVII, 393-406.

... 1987: "Comastas en Tartesos. En torno a la iconografía del vino y la danza simposíaca en la Península Ibérica”. Homenaje a Francisco R. Adrados. Vol. II, 683-696.

Olmos Romera, R. y Cabrera Bonet, P., 1980: "Un nuevo fragmento de Clitias en Huelva". Archivo Español de Arqueología, 53, 5-14.

Olmos Romera, R. y GARRIDO RoIZ J.P., 1982: “Cerámica griega en Huelva. Un informe preliminar”. Homenaje a Sáenz de Buruaga, Badajoz, 243-264.

PICAZO, M., 1984: La cerámica griega de Ullastret. Publicaciones Eventuales, n. 28. Barcelona.

Ripoll Perello, E. y Sanmarti Greco, E., 1978, "La expansión griega en la Península Ibérica”. II Congreso Internacional de Estudios sobre las culturas del Mediterráneo Occidental, 1975, Barcelona, 21-40.

Sanmarti Greco, E. y Gusi Jener, F., 1976: "Un kylix del Pintor Penthesilea, procedente del poblado ilercavon de El Puig (Benicaró, Castellón)”. Cuadernos de Prehistoria y Arqueología Castellonenses, 3, 205-218.

Santos Yanguas, N. y PiCazo, M., 1980: La colonización griega. Madrid.

SHEFTON, B. B., 1982: "Greeks and Greek imports in the South of the Iberian Peninsula. The archaeological evidence”. Phönizer im Westen. Madrider Beiträge, 8, 337-370-. 